\title{
Es que es muy difícil \\ Fremdsprachenpolitik und Fremdsprachenunterricht in Spanien
}

JesSCACL LüTH

M.A. Universität Hamburg

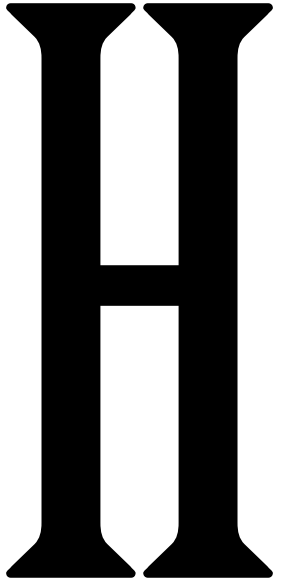

intergrund. Sätze wie Sí, claro que se aprende lenguas en el cole...ipero no se habla! oder Es que me da mucha vergüenza hablar... sind keine Seltenheit, wenn es in Spanien um Fremdsprachenunterricht geht. Warum ist das so? Und unter welchen Bedingungen werden in Spanien Fremdsprachen überhaupt unterrichtet? Insbesondere dieser Frage wollte ich im Rahmen meiner Abschlussarbeit an der Universität Hamburg ${ }^{1}$ nachgehen.

Vorwegzunehmen ist, dass die Arbeit keine statistisch repräsentative Untersuchung des Fremdsprachenunterrichts ist. Ihr Anspruch besteht darin, den institutionellen Rahmen von Fremdsprachenunterricht zu definieren und kritisch zu betrachten. Im Vergleich zu den politischen Rahmenbedingungen soll außerdem aufgezeigt werden, wie Fremdsprachenunterricht in der Praxis an Bildungseinrichtungen durchgeführt wird und wie die Situation von Menschen eingeschätzt wird, die in diesem Umfeld tätig sind. Der Fokus liegt dabei auf Bildungseinrichtungen, deren Sprachenangebot Deutsch beeinhaltet.

Das Forschungsdesign der Arbeit umfasst drei Teile:

- Eine umfassende Analyse der Gesetzestexte und Curricula, die 2008/2009 den Fremdsprachenunterricht an a) den Schulen und b) den staatlichen Sprachschulen (Escuelas Oficiales de Idiomas, EOI) determinierten.

- Eine Umfrage, die im November und Dezember 2009 online durchgeführt wurde. Ein Kriterium zur Auswahl der Bildungseinrichtungen war das Angebot von Deutsch. Insgesamt wurden 290 spanische Bildungseinrichtungen aus allen 17 autonomen Gemeinschaften (Comunidades Autónomas, CCAA) eingeladen, an der Umfrage teilzunehmen. 121 Bildungseinrichtungen aus 16 CCAA nahmen teil. Ohne Beteiligung blieb die CCAA Extremadura.

- Im Frühjahr 2010 wurden mehrere Interviews mit Lehrenden und Direktoren verschiedener Bildungseinrichtungen in Madrid und Umgebung geführt.

In diesem Artikel möchte ich die Ergebnisse meiner Arbeit zusammenfassen und die abschließenden Handlungsvorschläge darlegen, die aus meiner Sicht notwendig sind, um den Fremdsprachenunterricht und insbesondere den Deutschunterricht in Spanien langfristig zu stärken.

\section{Nationale Strukturen}

Das folgende Schema zeigt die verschiedenen nicht-universitären Bildungsbereiche in Spanien 2008/2009:²

\begin{tabular}{|c|c|c|c|}
\hline Bildungsbereich & $\begin{array}{l}\text { Deutsche } \\
\text { Entsprechung }\end{array}$ & $\begin{array}{l}\text { Alter der Lernenden } \\
\text { in Spanien }\end{array}$ & $\begin{array}{l}\text { Zeitliche } \\
\text { Unterteilung }\end{array}$ \\
\hline Educación Infantil (EI) & Kindergarten & 0 - 6 Jahre & $\begin{array}{l}1^{\circ}: 0-3 \text { Jahre } \\
2^{\circ}: 3-6 \text { Jahre }\end{array}$ \\
\hline Educación Primaria (EP) & Grundschule & 6 - 12 Jahre & $\begin{array}{l}1^{\circ}: 6 \text { - } 8 \text { Jahre } \\
2^{\circ}: 8 \text { - } 10 \text { Jahre } \\
3^{\circ}: 10 \text { - } 12 \text { Jahre }\end{array}$ \\
\hline Educación Secundaria & Sekundarstufe I & 12 - 16 Jahre & 4 einjährige Kurse \\
\hline Bachillerato (BA) & Sekundarstufe II & 16 - 18 Jahre & 2 einjährige Zyklen \\
\hline Alternativ: Formación & Berufsschule/Höhere & 16 - 18 Jahre & 1 oder 2 einjährige \\
\hline Profesional (FP) & Handelsschule & & Zyklen \\
\hline $\begin{array}{l}\text { Educación de Régimen } \\
\text { Especial: Escuelas } \\
\text { Oficiales de Idioma (EOI) }\end{array}$ & $\begin{array}{l}\text { olkshochschule, nur } \\
\text { Sprachunterricht }\end{array}$ & $\begin{array}{l}\text { Erwachsenenbildung, } \\
\text { ab } 16 \text { Jahre }\end{array}$ & beliebig \\
\hline
\end{tabular}

In der Arbeit werden Bildungseinrichtungen aller Bereiche mit Ausnahme der FP berücksichtigt. Der Grund dafür ist, dass eine Untersuchung des Fremdsprachenunterrichts innerhalb der FP wegen der unübersichtlichen Lage im Rahmen dieser Arbeit zu umfangreich erschien.

Alle oben genannten Bildungsbereiche unterstehen dem 2006 in Kraft getretenen nationalen Bildungsgesetz Ley Orgánica 2/2006, de 3 de Mayo, de Educación (LOE). Das Gesetz proklamiert drei Prinzipien, von denen vor allem das Dritte für den Fremdsprachenunterricht von Bedeutung ist, da es die Anlehnung der spanischen Bildungspolitik an die Bildungsrichtlinien der Europäischen Union beinhaltet:

El tercer principio que inspira esta Ley consiste en un compromiso decidido con los objetivos educativos planteados por la Unión Europea para los próximos años. El proceso de construcción europea está llevando a una cierta convergencia de los sistemas de educación y formación, que se ha traducido en el establecimiento de unos objetivos educativos comunes para este inicio del siglo XXI. (LOE 2006: 17160) 
Im Grundsatz zeigt sich hier ein potentieller Konflikt, der nicht nur auf Spanien sondern auf viele Mitgliedsstaaten der Europäischen Union zutrifft: die Vereinbarung europäischer und nationaler Bildungspolitik und damit einhergehender Interessen. In Spanien und einigen anderen Ländern lässt sich diese Problematik auf die regionale Ebene ausweiten, da sich die Bildungspolitik trotz des $L O E$ durch Dezentralisierung und Regionalisierung auszeichnet.

Doch inwiefern determiniert das LOE den Fremdsprachenunterricht? Das Erlernen einer ersten Fremdsprache wird in der $E P$ vorgegeben. Dabei ist festzuhalten, dass zum einen keine

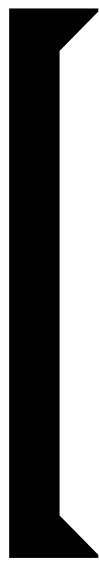

Hier wird ein Problem deutlich, welches sowohl fuir die erste als auch fuir eine mögliche zweite Fremdsprache zwischen allen Bildungsbereichen exisitiert: der Verlust der Kontinuität.

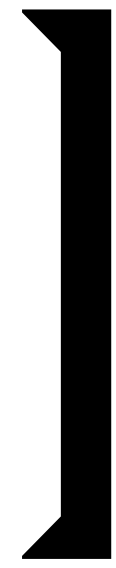

Präferenz gegenüber einer bestimmten Fremdsprache ausgesprochen wird und zum anderen alle Minderheitensprachen explizit von den Fremdsprachen abgegrenzt werden. Die Rolle der Minderheitensprachen wird im Zusammenhang mit dem Spanischunterricht wieder aufgegriffen. Diese Position wird dem Status der zusätzlichen Amtssprache ${ }^{3}$ in einigen CCAA gerecht, verkennt aber häufig die Ausgangssituation des einzelnen Individuums, das häufig zu Hause monolingual aufwächst und für das auch eine weitere Amtssprache eine Fremdsprache darstellt.

Eine erste Fremdsprache soll durchgehend in der ESO weitergelernt werden und ist auch im $B A$ Bestandteil des Pflichtfächerkanons. Für alle drei Bildungsbereiche werden allgemeine Zielsetzungen formuliert, als Beispiel soll hier die Formulierung für die ESO genügen: „Comprender y expresarse en una o más lenguas extranjeras de manera apropiada. " (LOE 2006: 17169) Was genau manera apropriada meint, wird nicht weiter erläutert.

In sogenannten Reales Decretos (RD), nationalen Gesetzeserweiterungen des $L O E$, werden ausführlichere Lernziele und Lerninhalte für den Fremdsprachenunterricht in den einzelnen Bildungsbereichen formuliert. Das RD 1630/2006 proklamiert bereits die Förderung einer positiven Einstellung gegenüber der Muttersprache und Fremdsprachen in der EI. Für die sechsjährige EP legt das $R D$ 1513/2006 einige allgemeine Lernziele fest und beschreibt Lerninhalte, die in vier Blöcke gegliedert werden: Sprechen und Hören, Lesen und Schreiben, Linguistik und Reflexion über den eigenen Lernprozess sowie Sozial- und Landeskunde. Alle Formulierungen werden allgemein gehalten und beziehen sich nicht explizit auf die Niveaustufen des GER. ${ }^{5}$ Interessanterweise gelten die selben Lernziele und Lerninhalte auch für eine zweite Fremdsprache, ohne dass mögliche Veränderungen der Bedingungen berücksichtigt werden. Zusätzlich zu Lernzielen und Lerninhalten wird auch die minimale Anzahl der Unterrichtseinheiten im RD 1513/2006 definiert. Demnach entspricht die Anzahl der Unterrichtseinheiten für die erste Fremdsprache im ersten Zyklus einem Drittel der Unterrichtseinheiten, die für den Spanischunterricht vorgesehen sind. In den folgenden zwei Zyklen nähert sich das Verhältnis an, trotzdem fallen noch doppelt so viele Unterrichtseinheiten auf den Spanischunterricht wie auf den Fremdsprachenunterricht.

Das RD 1631/2006 entspricht strukturell dem RD 1512/2006, allerdings werden gesondert Lernziele und Lerninhalte für eine zweite Fremdsprache, gegebenenfalls Deutsch, formuliert. Diese Formulierungen entkräften sich jedoch gewissermaßen selbst, indem zu Beginn darauf hingewiesen wird, dass sowohl Lernenden mit Vorkenntnissen als auch Lernenden ohne Vorkenntnisse die Teilnahme am Unterricht ermöglicht werden sollte. Die Lernziele und Lerninhalte müssten entsprechend von den Einrichtungen oder den Lehrenden modifiziert werden. Meines Erachtens ist Fremdsprachenunterricht in dieser Form praktisch nur schwer zu realisieren. Hier wird ein Problem deutlich, welches sowohl für die erste als auch für eine mögliche zweite Fremdsprache zwischen allen Bildungsbereichen existiert: der Verlust der Kontinuität. Lernende die in der EP bereits mit einer zweiten Fremdsprache begonnen haben, müssen die Möglichkeit haben, diese auch in der ESO fortführen zu können. Ein Neubeginn in der Fremdsprache bedeutet immense Rückschritte für diese Lernenden und untergräbt die Qualität des vorherigen Fremdsprachenunterrichts. Dies gilt natürlich auch für die Fortführung der ersten Fremdsprache. Da aber die Lernziele zu Abschluss der EP zwischen und teilweise auch innerhalb der CCAA divergieren, ist es schwer für Lehrende der ESO einen gemeinsamen Startpunkt zu finden. Wenn das Prinzip 'um so tiefer das Niveau, desto mehr Lernende kommen mit' lautet, kann diese Herangehensweise zu Demotivierung und Frustration jener Lernenden führen, die sich bereits gute Vorkenntnisse in der EP angeeignet haben.

Die Verteilung der Unterrichtseinheiten für Spanisch und die erste Fremdsprache nähern sich in der ESO viel stärker an als in den vorherigen Bereichen. Es entfallen im Durchschnitt in allen vier Kursen zwei bis drei Unterrichtseinheiten pro Woche auf den Fremdsprachenunterricht. Eine zweite Fremdsprache kann in den ersten drei Kursen angeboten werden, die Bedingungen werden dann von der jeweiligen CCAA bestimmt. Im vierten Kurs ist eine zweite Fremdsprache Teil des Wahlpflichtfächerkanons. Die propagierte Mindestanzahl von jährlich 70 Unterrichtseinheiten entspräche einer bis zwei Unterrichtseinheiten pro Woche im vierten Kurs. In Hinblick darauf, dass viele Lernende erst jetzt mit einer zweiten Fremdsprache beginnen, die ESO aber nach diesem Kurs abgeschlossen wird, ist zu bezweifeln, dass die Lernenden von diesem Angebot besonders profitieren werden.

Denn das bereits erwähnte Problem der Kontinuität besteht auch zwischen der ESO und dem BA. Im RD 1467/2007 wird zwar eine erste Fremdsprache als Pflichtfach vorgegeben, allerdings bleibt unklar, auf welchen Vorkenntnissen die Lehrenden ihren Unterricht aufbauen können und welches Niveau die Lernenden nach Abschluss erreichen sollen. Auf eine zweite Fremdsprache wird nicht eingegangen, da sie nur als Wahl- oder Wahl- 
pflichtfach innerhalb einer Spezialisierung ${ }^{6}$ angeboten wird und dem entsprechend für das Ausformulieren von Lernzielen und Lerninhalten die CCAA oder die einzelnen Bildungseinrichtungen zuständig sind.

Zusammenfassend ist festzuhalten, dass die Konkretisierung von Lernzielen und Lerninhalten in allen Bildungsbereichen des Vorschul- und Schulwesens den CCAA bzw. den Bildungseinrichtungen überlassen wird. Das $L O E$ und die zugehörigen $R D$ legen vage formulierte Lernziele fest, aber eine Kontinuität zwischen den einzelnen Bereichen wird nur in Ansätzen deutlich. Des Weiteren legt das LOE nur das Erlernen von einer Fremdsprache verpflichtend fest. Ab der ESO sollen zwar alle Einrichtungen eine zweite Fremdsprache anbieten, allerdings obliegt es den CCAA ihren Status zu bestimmen, d. h. sie kann als Wahl-, Wahlpflicht- oder Pflichtfach eingeführt werden. Das Erlernen einer dritten Fremdsprache in der ESO oder im $B A$ wird nicht diskutiert.

Das LOE determiniert ebenfalls den Fremdsprachenunterricht an den EOI. Dort werden drei Niveaustufen festgelegt: Básico, Intermedio und Avanzado. Hier können neben Fremdsprachen zum Teil auch die weiteren Amtssprachen gelernt werden. In der Gesetzeserweiterung Real Decreto 1629/2006 werden die Niveaustufen dem GERS angepasst und kommen zu einem teilweise erstaunlichen Ergebnis:

- Zum Abschluss des Niveaus Básico sollen Lernende das Niveau A2 erreicht haben.

- Zum Abschluss des Niveaus Intermedio sollen Lernende das Niveau B1 erreicht haben.

- Zum Abschluss des Niveaus Avanzado sollen Lernende das Niveau B2 erreicht haben.

Erstaunlich ist dieses Ergebnis insofern, als dass keine der spanischen Niveaustufen zu der Stufe C1 oder C2 des GER hinführt. In einer Zusatzbemerkung wird kurz darauf eingegangen:

RD 1629/2006 Disposición adicional segunda:

Las Escuelas Oficiales de Idiomas podrán, en los términos que dispongan las respectivas Administraciones educativas, organizar e impartir cursos especializados para el perfeccionamiento de competencias en idiomas tanto en los niveles básico, intermedio y avanzado como en los niveles $\mathrm{C} 1$ y C2 del Consejo de Europa según estos niveles se definen en el Marco Común Europeo de Referencia para Lenguas. (RD 1629/2006: 466)

Es bleibt zu hinterfragen, ob eine derartige Modifizierung der Niveaustufen auf nationaler Ebene sinnvoll ist.

Die Universitäten unterstehen dem Ley Orgánica de Universidades (LOU), das 2001 in Kraft trat. Bezüglich des Fächerangebots unterliegen die Universitäten keinen nationalen Vorschriften. Dem entsprechend wird das Gesetz an dieser Stelle nicht weiter erörtert.

\section{Regionale Strukturen}

Was die nationalen Strukturen erahnen lassen, wird auf der Ebene der regionalen Strukturen deutlicher: die CCAA bear- beiten die nationalen Vorgaben auf verschiedene Arten und Weisen. Heraus kommen divergierende Standards, die letzten Endes zum einen den Vergleich von Bildungseinrichtungen innerhalb und zwischen den CCAA, zum anderen die Mobilität von Lernenden und Lehrenden erschweren.

Im Folgenden sollen die auffälligsten Merkmale, die sich aus der Analyse der regionalen Gesetzgebungen (Curricula) ergeben haben, erläutert werden:

In ihrer Struktur ähneln sich die meisten untersuchten Curricula: Sie bestehen grundsätzlich aus Richtlinien und einem Lehrplan. Für jeden Bildungsbereich wird in der Regel ein separates Curriculum entwickelt. Die CCAA des Baskenlandes hat im Gegensatz dazu jedoch ein gemeinsames Curriculum für die $E P$ und die ESO, d. h. für jene Bildungsbereiche, die die Schulpflicht ausmachen. Für die Wahrung der Kontinuität zwischen den Bereichen könnte das einen guten alternativen Ansatz darstellen.

In der EI wird in allen Curricula der Kontakt mit einer Fremdsprache als Begegnungssprache gefördert. In einigen CCAA muss diese Fremdsprache ebenso wie die erste Fremdsprache in der EP Englisch sein, in anderen CCAA wird die Wahl der ersten Fremdsprache den Bildungseinrichtungen freigestellt. In den meisten Fällen werden sich diese dann wohl trotzdem für Englisch entscheiden, es eröffnet aber auch andere Möglichkeiten: So gibt es beispielsweise in Extremadura Schulen, die als erste Fremdsprache Portugiesisch anbieten. Anders verhält es sich in den meisten CCAA, in denen eine weitere Amtssprache neben dem Spanischen besteht. In diesen Regionen erfolgt häufig eine durch das Curriculum vorgegebene bilinguale Ausbildung von der EI an, auch wenn bilingual in diesem Zusammenhang nicht zwingend bedeutet, dass beide Sprachen für jedes Individuum gleichwertig sind.

Die Anzahl der Unterrichtseinheiten für eine erste Fremdsprache nimmt in der Regel in allen Curricula im Verlauf der $E P \mathrm{zu}$ und bleibt in den folgenden Bildungsbereichen relativ hoch. Nur in wenigen CCAA wird die Anzahl in höheren Bildungsbereichen gesetzlich reduziert, um Zeit für neue Fächer, z. B. die Einführung einer zweiten Fremdsprache, zu sichern. In allen untersuchten CCAA wird eine zweite Fremdsprache als Wahl- oder Wahlpflichtfach und überwiegend mit einer geringeren Anzahl von Unterrichtseinheiten als die erste Fremdsprache angeboten. Dabei wäre es doch nachvollziehbar, ein neues Fach mit einer relativ hohen Anzahl an Unterrichtseinheiten auszustatten, insbesondere um eine zügige Lernprogression $\mathrm{zu}$ fördern und so die Motivation der Lernenden zu erhalten. So sind im Curriculum von Katalonien die erste und die zweite Fremdsprache in der ESO gleichberechtigt und werden mit je drei Unterrichtseinheiten pro Woche unterrichtet.

In keinem der Curricula für das $B A$ wird eine zweite Fremdsprache gefordert. Erstaunlicherweise wird für die Spezialisierung in Human- und Sozialwissenschaften eine zweite Fremdsprache lediglich als Wahlfach angeboten, während Griechisch und Latein im Kanon der Wahlpflichtfächer stehen. Hier ist doch fraglich, warum bisher keine Modernisierung zu Gunsten moderner Fremdsprachen erfolgt ist, sei es auf regionaler oder nationaler Ebene.

Die Formulierungen von Richtlinien und Lehrplänen differenzieren bezüglich ihrer Ausführlichkeit und ihrer Gliederung in Teilbereiche. Zum Teil bestehen diese Differenzen bereits zwi- 
schen den einzelnen Bildungsbereichen innerhalb einer CCAA. Unter solchen Umständen ist es dem entsprechend schwieriger, eine durchgängige Kontinuität zwischen den Curricula zu etablieren bzw. zu verdeutlichen. Einige der CCAA formulieren sprachspezifische Lerninhalte für verschiedene Sprachen, z. B. Kastilien und León, andere haben ein allgemeingültiges Curriculum für den Fremdsprachenunterricht, unabhängig von der Sprache, die angeboten wird. Ähnliche Differenzen lassen sich in Bezug auf die zweite Fremdsprache ausmachen. Während einige CCAA sprachspezifische Curricula für mögliche zweite Fremdsprachen formulieren, wird in anderen CCAA auf das Curriculum der ersten Fremdsprache verwiesen. Die meisten Curricula geben an, sich am GERS zu orientieren, doch lediglich zwei CCAA proklamieren explizite Lernziele nach dem GERS. Im Baskenland sollen demnach alle Lernenden zum Abschluss der ESO Kenntnisse des Niveau B1 in der ersten Fremdsprache erworben haben. Diese sollen im BA bis auf das Niveau B2 ausgeweitet werden. Auch im Curriculum von Andalusien wird ein klares Lernziel für die Lernenden des $B A$ formuliert: Sie sollen in der ersten Fremdsprache Kenntnisse des Niveau B1 erwerben.

Auf die bereits angesprochene Forderung durch die $R D$, dass

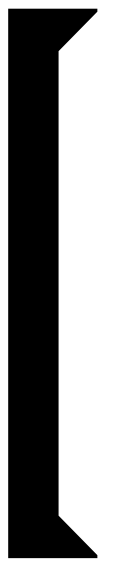

Aus den Interviews geht die Aussage hervor, dass sich die gevwinsshte europäische Mehrsprachigkeit fuir die meisten Lernenden in Spanien als Zweisprachigkeit entpuppt. Dienationalen Autoritätenwerden kitisisert, dieNotwendigkeiteinerverpflichtenden zweiten Fremdsprache zu verkennen.

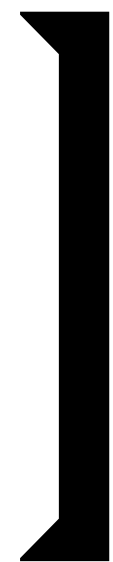

der Fremdsprachenunterricht Lernenden mit und ohne Vorkenntnissen zugänglich sein soll, reagieren die meisten regionalen Curricula verhalten und übergeben die Anpassung von Lernzielen und Lerninhalten an die einzelnen Bildungseinrichtungen. Lediglich Andalusien zeigt eine alternative Herangehensweise und hat das Curriculum geteilt, so dass es Lernziele und Lerninhalte für Lernende mit und ohne Vorkenntnisse gibt. Ob sich diese Art von Curriculum in der Praxis als vorteilhaft erweist, bleibt fraglich, doch zumindest wird hier von Seiten der regionalen Gesetzgeber auf die Problematik hingewiesen und versucht, den Bildungseinrichtungen einen Lösungsvorschlag anzubieten.

Die untersuchten Curricula für die EOI wirken insgesamt alle ähnlich strukturiert und orientieren sich explizit am GERS. Auch wenn die Formulierungen teilweise voneinander abweichen und die angegebene Kursdauer für einzelne Niveaustufen variiert, wird durch die gemeinsamen Lernziele nach dem GERS eine gewisse Kontinuität geschaffen. Das Baskenland ist die einzige CCAA, die in ihrer regionalen Gesetzgebung zusätzlich die Stufe C1 einführt.

\section{Fremdsprachenunterricht in der Praxis}

Im Folgenden sollen die Ergebnisse der Online-Umfrage für die Bildungsbereiche Schulen (EI-BA), Universitäten und EOI dargelegt werden. Diese werden von Tendenzen ergänzt, die sich aus der Analyse der Interviews ergeben haben.

Wie aus den Curricula hervorgehend, bestätigt auch die Umfrage, dass in allen untersuchten CCAA die erste Fremdsprache entweder in der $E I$ oder in der $E P$ eingeführt und mit einer hohen Anzahl an Unterrichtseinheiten unterrichtet wird. Das Kriterium, dass alle untersuchten Einrichtungen Deutsch im Angebot haben müssen, führt hier zu einer interessanten Entwicklung: Deutsch als erste Fremdsprache dominiert vor allem an privaten ${ }^{8}$ Schulen, Englisch als erste Fremdsprache an staatlichen Schulen. Dem entsprechend ist Deutsch an staatlichen Schulen überwiegend zweite oder weitere Fremdsprache, wenn diese denn überhaupt angeboten wird.

Während eine zweite Fremdsprache in der ESO und im $B A$ zumindest als Wahlfach angeboten werden muss, zeigt die Umfrage, dass weniger als die Hälfte aller in Frage kommenden Bildungseinrichtungen zusätzlich das Erlernen einer dritten modernen Fremdsprache anbieten. Außerdem wird deutlich, dass die Einführung einer zweiten Fremdsprache bezüglich des Zeitpunktes und der Anzahl der vorgesehenen Unterrichtseinheiten uneinheitlich erfolgt. Die Analyse der regionalen Gesetzestexte hat gezeigt, dass das Wahren einer inhaltlichen Kontinuität problematisch sein könnte, auch wenn eine institutionelle Kontinuität gegeben ist, $\mathrm{d}$. h. wenn festgelegt wird, dass eine erste Fremdsprache bis zur ESO oder ggf. bis zum $B A$ gelernt werden muss. Die unterschiedlichen Bedingungen unter denen eine zweite Fremdsprache eingeführt wird, lassen vermuten, dass in diesem Fall sowohl die inhaltliche als auch die institutionelle Kontinuität fehlen.

Aus den Interviews geht die Aussage hervor, dass sich die gewünschte europäische Mehrsprachigkeit für die meisten Lernenden in Spanien als Zweisprachigkeit entpuppt. Die nationalen Autoritäten werden kritisiert, die Notwendigkeit einer verpflichtenden zweiten Fremdsprache zu verkennen. Außerdem scheinen sich einige CCAA auf die Förderung einer bestimmten Sprache zu konzentrieren: so fokussiere Madrid sowie viele andere CCAA die bilinguale spanisch-englische Bildung. Und in der Tat lässt das Fremdsprachenangebot an den untersuchten Einrichtungen nur in Ansätzen eine aktive Förderung der Mehrsprachigkeit erkennen. Obwohl Nachbarlandssprache, wird Portugiesisch an keiner der untersuchten Einrichtung angeboten. Einige Einrichtungen bieten Italienisch, eine Chinesisch an. Obwohl den meisten Einrichtungen vom Gesetz freigestellt wird, welche Fremdsprachen sie anbieten, konzentriert sich die Mehrheit auf Englisch, Französisch und Deutsch.

Es mag Stimmen geben, die bilingualen Unterricht als Einschränkung für den Fremdsprachenunterricht sehen. Die Analyse der Curricula und die Umfrage zeigen allerdings eher gegenteilige Tendenzen. Insbesondere die Curricula aus Katalonien und dem Baskenland zeigen, dass eine bilinguale Unterrichtssituation nicht zwingend zu einer Reduzierung des Fremdsprachenangebots oder dessen Rahmenbedingungen führen muss. Aus den Interviews geht für das Vorschul- und Schulwesen insgesamt die Tendenz hervor, dass eine rege Nachfrage nach Deutsch vorhanden sei. In den Ballungszentren um die spanischen Großstädte könne 
der Nachfrage teilweise nicht mehr nachgekommen werden.

An den Universitäten zeigt sich eine starke Tendenz weg von eigenständigen deutschsprachigen Studiengängen hin zu Deutsch als Begleitstudiengang. Im Zuge der Bologna-Reform haben vor allem zusammenfassende Studiengänge wie Moderne Sprachen oder praxisorientierte Studiengänge wie Übersetzungswissenschaften profitiert, innerhalb derer Deutsch gewählt werden kann. In den Interviews wird das Anliegen der EU, die Effizienz der europäischen Hochschulen zu steigern, grundsätzlich positiv gesehen, allerdings wird kritisiert, dass insbesondere Philologien darunter zu leiden hätten. Germanistik wurde zum Zeitpunkt der Umfrage an sieben Universitäten angeboten, Tendenz sinkend.

Die Umfrage zeigt, dass der Anspruch in Bezug auf die zu erreichenden Sprachniveaus an den Universitäten zu sinken scheint. Während für Germanisten noch das Erreichen der Niveaustufe C nach dem GERS angestrebt wird, ist die in den Modernen Sprachen häufigste genannte Niveaustufe B1. Es könnte eine Entwicklung sein, die einen Qualitätsverlust als Folge von Lehrkräftemangel bei steigender Studierendenanzahl mit sich bringt. Der tatsächliche Grund bleibt hier allerdings offen. Des Weiteren ist insbesondere im Bereich Tourismus eine zunehmende Bedeutung von Deutsch zu erkennen, aber auch in anderen Studiengängen, z. B. Jura und Wirtschaftswissenschaften.

Die Tatsache, dass die Anzahl der EOI in den letzten zwei Jahrzehnten kontinuierlich gestiegen ist, zeigt, dass es in Spanien - vielleicht entgegen vieler Erwartungen - eine Nachfrage nach Fremdsprachen zu geben scheint. Den Angaben aus der Umfrage zu Folge bleibt Englisch die mit Abstand beliebteste Sprache vor Französisch und Deutsch. Aus den Interviews geht hervor, dass insbesondere die Nachfrage nach Deutsch zum Zeitpunkt der Untersuchung stagniere. Kritisch gesehen wird in den Interviews das Fehlen der Niveaustufe C nach dem GERS sowie das Fehlen konkretisierender Richtlinien zur Umsetzung nationaler Vorgaben, um die Mobilität von Lernenden und Lehrenden an den EOI zu erleichtern. Interessant sind außerdem die Umfrageergebnisse zur Verteilung der Lernenden nach Alter, denn an den meisten Einrichtungen liegt der Altersdurchschnitt der Lernenden zwischen 21 und 35 Jahren. Bei jüngeren Lernenden, die quasi direkt aus der Schule kommen, muss man sich die Frage stellen, inwiefern der schulische Fremdsprachenunterricht unzureichend war, so dass Lernende jetzt eine Weiterbildung an der EOI anstreben. Die Umfrage zeigt außerdem, dass es Probleme gibt, Deutschlernende über das Anfängerniveau hinaus zu halten. Während es im Englischen einen hohen Prozentsatz an Lernenden gibt, der sich auf den Niveaustufen B und ggf. $\mathrm{C}$ weiterbildet, beenden viele Lernende ihren Deutsch- oder Französischunterricht nach der Niveaustufe A. Hier könnte die subjektiv empfundene Schwierigkeit des Deutschen eine Rolle spielen. Die Interviews zeigen aber, dass diese Schwierigkeit auch Potential bergen kann, denn Deutsch zu lernen bedeutet auch, die Willensstärke zu zeigen, sich mit dieser Sprache auseinanderzusetzen. Dies könnte ein alternativer Ansatz sein, dem Vorurteil der „Unmöglichkeit des Deutschlernens“ entgegenzuwirken.

Ein zentraler Aspekt, der sich aus den Interviews entwickelte und für alle Bildungsbereiche gleichermaßen relevant ist, ist die Lehreraus- und Fortbildung sowie die finanziellen Mittel um diese Sektoren auszubauen und zu fördern. Positiv bewertet werden Veränderungen im Bereich der Lehrerausbildung, allerdings seien diese Veränderungen noch auszuweiten, z. B. auf andere Bildungsbereiche wie die Universität. Eher kritisch wird dagegen das Angebot zur Lehrerfortbildung gesehen. Hier wird eine generelle Ausweitung des Angebots, sowie eine Spezifizierung nach Sprachen und nach Zielgruppen gefordert.

\section{Forschungsfragen und Handlungsvorschläge}

In Zusammenhang mit dieser Arbeit und den Forschungsbereichen Fremdsprachenunterricht und Fremdsprachenpolitik, lassen sich u. a. folgende Forschungsfragen formulieren:

- Wie kann eine Zusammenarbeit zwischen Politik und Wissenschaft für die Verbesserung des Fremdsprachenunterrichts aussehen?

- Welche Formen kann Fremdsprachenpolitik in politisch dezentralisierten Ländern haben?

- Inwiefern lässt sich internationale, nationale und regionale Fremdsprachenpolitik kombinieren?

- Inwiefern können historische Ereignisse oder Entwicklungen zu Hemmungen gegenüber Fremdsprachenunterricht führen

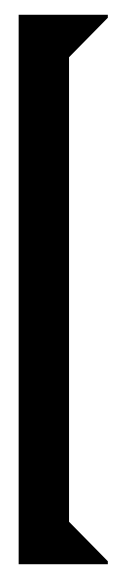

Außserdem könnten sich Vertreter verschiedener Sprachen,Bildungseinrichtungen und CCAAaus-

tauschen und sich zusammen für eine offiziell höhere Anerkennung von Fremdsprachen einsetzen. VoneinemhöherenAnsehenallerFremdsprachen profitiert am Ende auch jede einzelne.

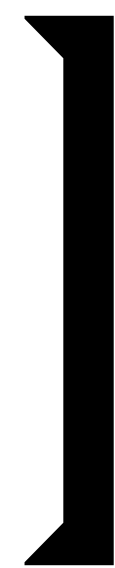

und wie kann ihnen entgegengewirkt werden?

- Wie lässt sich die Nachfrage nach Sprachen in der Bevölkerung adäquat messen und wie kann flexibler darauf reagiert werden?

- Wie lässt sich die „Schwierigkeit“ einer Sprache messen?

- Welche Rolle können weitere Amtssprachen im Fremdsprachenunterricht spielen?

Es ist evident, dass sich diese Liste weiter fortsetzen ließe und weitere Forschung notwendig ist, um Fremdsprachenunterricht und Fremdsprachenpolitik in ihrer gesamten Komplexität adäquat beschreiben zu können.

Basierend auf den vorgelegten Ergebnissen lassen sich abschließend einige Handlungsvorschläge formulieren:

- Eine zweite Fremdsprache sollte in der EP und in der ESO verpflichtend eingeführt werden.

- Eine zweite moderne Fremdsprache sollte Teil des Wahlpflichtfächerkanons in der $B A$ Spezialisierung Human- und Sozial- 
wissenschaften werden.

- Die bisherigen minimalen Standards der RD sollten dem GERS angepasst werden und unmissverständliche Zielsetzungen proklamieren.

- Ein Leitfaden zur Umsetzung des LOE, insbesondere zur Erstellung und Weiterentwicklung fachspezifischer Curricula sollte erstellt werden; dieser Leitfaden soll verschiedene alternative Umsetzungsmöglichkeiten aufzeigen.

- In Bezug auf die EOI sollte die höchste Niveaustufe an den GERS angepasst werden und damit auf das Niveau C angehoben werden.

- Regionale Fremdsprachencurricula sollten im Hinblick auf ihre Kontinuität überprüft und ggf. überarbeitet werden.

- Die Lehrerausbildung sollte nicht nur für bestimmte, sondern für alle Bereiche verbessert werden. Auch hier gilt das Prinzip der Kontinuität.

- Das Fortbildungsangebot sollte ausgeweitet und spezifiziert werden.

- Das Bewusstsein der Bevölkerung für die Bedeutung von Fremdsprachen in der Gesellschaft und von Fremdsprachenunterricht für die Lernenden sollte geschärft werden.

- Das Vorurteil, Deutsch sei „unmöglich“ zu lernen, sollte abgebaut werden.
Viele dieser Handlungsvorschläge können nur mit Hilfe finanzieller Unterstützung und demnach in kleinen Schritten umgesetzt werden. Alle Initiativen sind auf engagierte Menschen angewiesen, die sich für Fremdsprachen begeistern. In Bezug auf die letzten beiden Punkte ist es m. E. wichtig, weniger die Vorurteile und Schwierigkeiten sondern die Attraktivität fremder Sprachen herauszuarbeiten und anzuerkennen. In Spanien entsteht historisch bedingt erst seit wenigen Jahrzehnten ein Bewusstsein für die Bedeutung fremder Sprachen. Englisch gewinnt weiterhin an Boden auf Grund seiner Rolle als Weltsprache und Französisch wird insbesondere durch die EU-Institutionen in seiner Wichtigkeit bestärkt. Deutsch wird häufig mit der Wirtschaftskraft Deutschlands verbunden, ein Faktor, der durch die derzeitige Finanzkrise in Europa noch bedeutender werden könnte. Im Sinne der europäischen Bildungspolitik, an der sich das spanische Bildungsgesetz orientieren möchte, sollten aber auch andere Sprachen gleichermaßen von Seiten nationaler und regionaler Autoritäten gefördert werden. Außerdem könnten sich Vertreter verschiedener Sprachen, Bildungseinrichtungen und CCAA austauschen und sich zusammen für eine offiziell höhere Anerkennung von Fremdsprachen einsetzen. Von einem höheren Ansehen aller Fremdsprachen profitiert am Ende auch jede einzelne.
1. Jessica Lüth:Deutschunterrichtanspanischen Bildungseinrichtungen - eine Bestandsanalyse unter Berücksichtigung fremdsprachenpolitischer Aspekte. Wissenschaftliche Hausarbeit zur Erlangung des akademischen Grades Magistra Artium, Universität Hamburg. Für weitere Informationen: jessica.lueth@kcl.ac.uk

2. Tabelle: Jessica Lüth

3. Jenach Bildungsbereichen werden die Bereiche in Zyklen (ciclos) oder Kurse (cursos) unterteilt. Die Länge variiert von Bereich zu Bereich.

4. span. lenguas co-oficiales: Es handelt sich in verschiedenen CCAA um Katalanisch und/oder dazugehörige Variationen, Baskisch und/oder Galicisch.

5. GemeinsamerEuropäischer Referenzrahmen für Sprachen: Lernen, lehren, beurteilen.

6. Im BA können die Lernenden zwischen drei Spezialisierungen wählen: a) Kunst, b) Wissenschaften und Technologie und c) Human- und Sozialwissenschaften.

7. Im Rahmen der Arbeit wurden folgende regionale Gesetzestexte untersucht: für Andalusien, Aragón, die Kanarischen Inseln, Kantabrien, Katalonien, Kastilien und León, Madrid und das Baskenland Curricula für die Schulen und die EOI. Für Asturien, die Balearen, Kastilien und La Mancha, Galizien, Murcia, Navarra und Valencia ausschließlich die Curricula für die EOI.

Für weitere Informationen bzw. vollständige Bibliographie: jessica.lueth@kcl.ac.uk

8. Privat- oder staatlich anerkannte Privatschulen werden unter dem Begriff private Schulen zusammengefasst.
Bibliographie zum Thema Sprachenpolitik und Deutsch in Spanien:

Acosta, Luis A. (2001), Germanistik und DeutschandenspanischenUniversitäten: Institute,Lehrkräfteund Studenten.Stand 2001, BOA, Asociación Madrileña de Germanistas, Madrid

Balzer, B., Benito Rey, M., Caesar, S. et al. (Hrsg.) (2008), Germanistik und Deutschunterricht in Spanien. Deutsch als Fremdsprache und Linguistik. Germanística y Enseñanza del Alemán en España. Alemán como Lengua Extranjera y Lingüística, Vol. I Editorial Idiomas, Madrid.

Bausch, K.-R., Christ, H., Krumm, H.J. (Hrsg.) (2007), Handbuch Fremdsprachenunterricht, 5. Aufl. Francke, Tübingen

Bausch, K.-R. (2007), "Funktionen des Curriculums für das Lehren und Lernen fremder Sprachen", in Bausch et al. (Hrsg.) (2007), Handbuch, S. 111-116.

Bosch Roig, G. (1999), „Sprachenpolitik und Deutschunterricht in Spanien. Ein Beitrag zu einem empirisch begründeten sprachenpolitischen Konzept“ (Diss.) Bielefeld 1999. http://bieson.ub.uni-bielefeld.de/volltexte/2003/308/pdf/0019.pdf

Christ, H. ( 2007), ,Sprachenpolitik und das Lehren und Lernen fremder Sprachen",InBausch,K.-R./etal.(Hrsg.) (2007), Handbuch, S. 102-110.

Dräxler,H.D. (2006), ,Deutsch in Spanien, offensichtlich sinnvoll, aber scheinbar nicht vermittelbar. Sprachenpolitische Fakten und Überlegungen.", in AGA und FAGE (eds.), Magazin Nr. 12/2006, S. 10-13.

Ehlers, Ch. (1996), „Der Deutschunterricht in der Escuela Oficial de Idiomas in Andalusien. Bestandauf- nahme und Zukunftsaussichten", in AGA (Hrsg.) (1996), Magazin Nr. 1, S. 46-54.

Europäische Kommission(Hrsg.) (2008), Viele Sprachen für ein Europa. Sprachen in der europäischen Union. Brüssel. http://ec.europa.eu/publications/ booklets/move/74/de.pdf

Eurydice (2008) (Hrsg.), Schlüsselzablen zum Sprachenlernen an den Schulen in Europa, Brüssel.

http://eacea.ec.europa.eu/education/eurydice/documents/key_data_series/095DE.pdf

Eurydice (2009) (Hrsg.), Hochschulbildung in Europa 2009: Entwicklungen im Rabmen des Bologna-Prozesses, Brüssel.

http://eacea.ec.europa.eu/education/eurydice/documents/thematic_reports/099DE.pdf

Goethe-Institut Madrid (Hrsg.)( 2007), Deutschlernen in Spanien, Madrid. http:/www.goethe.de/mmo/priv/29608 08-STANDARD.pdf (08.07.2009)

Goethe-Institut Madrid (Hrsg.)( 2007), Deutschunterrichtanallgemein bildenden Schulen und staatlichen Sprachschulen in Spanien. Auswertung der Statistik des spanischen Erziehungsund Wissenschaftsministeriums für das Schuljahr 2005/2006, Madrid http://www.goethe.de/mmo/priv/ 2960804-STANDARD.pdf

Huguet, Á., Lapresta, C., Chireac, S.M. (2009), „Bilinguale Erziehung im Mehrsprachenstaat Spanien“, in Koskensalo, A. et al. (Hrsg.), The role of language in culture and education. Sprache als kulturelle Herausforderung, LIT Verlag, Berlin und Münster, S. 57-87.

Orduña, J. (2006), „Aus der Peripherie des Netzwerkes. Inlands-und Auslandsgermanistik aus spanischer Sicht", in
DaF, Nr. 43, Heft 3.2006, S.131-137. Siguan, M. (2007). , Die deutsche Sprache in Spanien", in Ammon, U., Globale Zukunftsperspektiven für internationale Germanistik, Jg. 39/2 Jahrbuch für Internationale Germanistik, S. 51-61.

Siguán, M. (2008), „Spanien “, in Institut für Deutsche Sprache IDS (Hrsg.), Germanistik und Deutschunterricht in 11 Ländern, IDS, Mannheim.

\section{Gesetztestexte:}

Ley Orgánica 2/2006, de 3 de mayo, de Educación. In: Boletín Oficial deEspaña (BOE) Nr. 106, 2006, S. 17158 17207.

Ministerio de Educación y Ciencias:

Real Decreto 1630/2006, de 29 de diciembre, por el que se establecen las enseñanzas mínimas del segundo ciclo de Educación Infantil. In: Boletín Oficial de España (BOE) Nr. 4, 2007, S. 474-482.

Real Decreto 1513/2006, de 7 de diciembre, por el que se establecen las enseñanzas mínimas de la Educación Primaria. In: Boletín Oficial de España (BOE) Nr. 293, 2006, S. 43053- 43102.

Real Decreto 1631/2006, de 29 de diciembre, por el que se establece las enseñanzas mínimas correspondientes a la Educación Secundaria Obligatoria. In: Boletín Oficial de España (BOE) Nr. 5, 2007, S. 677-773.

Real Decreto 1467/2007, de 2 de noviembre, por el que se establece la estructura del Bachillerato y se fijan sus enseñanzas mínimas. In: Boletín Oficial de España (BOE) Nr. 266, 2007, S. 45381- 45477. 